\title{
The spectra of IceCube neutrino candidate sources - I. Optical spectroscopy of blazars
}

\author{
Simona Paiano ${ }^{1,2,3 \star}$, Renato Falomo ${ }^{4}$, Aldo Treves ${ }^{5,6}$, Paolo Padovani ${ }^{7,8}$, \\ Paolo Giommi ${ }^{9,10,11}$, Riccardo Scarpa ${ }^{12,13}$ \\ ${ }^{1}$ INAF - Osservatorio Astronomico di Roma, via Frascati 33, I-00040, Monteporzio Catone, Italy \\ ${ }^{2}$ INAF - IASF Milano, via Corti 12, I-20133, Milano, Italy \\ ${ }^{3}$ INAF - IASF Palermo, via Ugo La Malfa, 153, I-90146, Palermo, Italy \\ ${ }^{4}$ INAF - Osservatorio Astronomico di Padova, vicolo dell'Osservatorio 5, I-35122, Padova, Italy \\ ${ }^{5}$ Universita' dell'Insubria, via Valeggio, 22100, Como, Italy \\ ${ }^{6}$ INAF - Osservatorio Astronomico di Brera, via Bianchi 46, I-23807, Merate (Lecco), Italy \\ ${ }^{7}$ European Southern Observatory, Karl-Schwarzschild-Str. 2, D-85748 Garching bei München, Germany \\ ${ }^{8}$ Associated to INAF - Osservatorio di Astrofisica e Scienza dello Spazio, Via Piero Gobetti 93/3, I-40129 Bologna, Italy \\ ${ }^{9}$ Institute for Advanced Study, Technische Universität München, Lichtenbergstrasse 2a, D-85748 Garching bei München, Germany \\ ${ }^{10}$ Associated to Agenzia Spaziale Italiana, ASI, via del Politecnico s.n.c., I-00133 Roma Italy \\ ${ }^{11}$ ICRANet, Piazzale della Repubblica 10, I-65122, Pescara, Italy \\ ${ }^{12}$ Instituto de Astrofisica de Canarias, C/O Via Lactea, s/n E38205 - La Laguna (Tenerife) - SPAIN \\ ${ }^{13}$ Universidad de La Laguna, Dpto. Astrofsica, s/n E-38206 La Laguna (Tenerife) - SPAIN
}

Received: 13 April 2021; Accepted: 13 April 2021

\section{ABSTRACT}

There is mounting evidence that ultra-energetic neutrinos of astrophysical origin may be associated with blazars. Here we investigate a unique sample of 47 blazars, $~ 20$ of which could be new neutrino sources. In particular, we focus on 17 objects of yet unknown redshift, for which we present optical spectroscopy secured at the Gran Telescopio Canarias and the ESO Very Large Telescope. We find all sources but one (a quasar) to be BL Lac objects. For nine targets we are able to determine the redshift $(0.09<\mathrm{z}<1.6)$, while for the others we set a lower limit on it, based on either the robust detection of intervening absorption systems or on an estimation derived from the absence of spectral signatures of the host galaxy. In some spectra we detect forbidden and semi-forbidden emission lines with luminosities in the range $10^{40}-10^{41} \mathrm{erg} \mathrm{s}^{-1}$. We also report on the spectroscopy of seven blazars possibly associated with energetic neutrinos that partially meet the criteria of our sample and are discussed in the Appendix. These results represent the starting point of our investigation into the real nature of these objects and their likelihood of being neutrino emitters.

Key words: galaxies: active and redshifts — BL Lacertae objects: general — gamma-rays: galaxies — neutrino

\section{INTRODUCTION}

The IceCube Neutrino Observatory at the South Pole ${ }^{1}$ has detected tens of high-energy neutrinos of likely astrophysical and extragalactic origin reaching beyond the $\mathrm{PeV}\left(10^{15} \mathrm{eV}\right)$ range (e.g. Schneider 2019; Stettner 2019; Aartsen et al. 2020, and references therein). These neutrinos are thought to be generated when very high-energy (VHE) cosmic rays (CRs) interact with matter or radiation creating charged and neutral mesons, which then decay into neutrinos, $\gamma$ rays, and other particles. At variance with CRs, neutrinos and $\gamma$ rays are the "messengers" that can travel cosmological distances undeflected. However, $\gamma$-ray spectra are softened by pair-production interactions with the extragalactic background light (EBL) at $E \gtrsim$ $100 \mathrm{GeV}$ in an energy- and redshift-dependent fashion (see, e.g.,

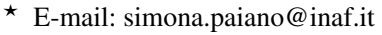

1 http://icecube.wisc.edu
}

Biteau et al. 2020; Cherenkov Telescope Array Consortium 2020, and references therein). This means that the extragalactic photon sky is almost completely dark at the energies sampled by IceCube $(\gtrsim 60$ $\mathrm{TeV}$ ). Therefore, only neutrinos can provide information on the VHE physical processes that generated them.

So far, only one astronomical object has been significantly associated (at the $3-3.5 \sigma$ level) in space and time with IceCube neutrinos, i.e., the powerful blazar TXS 0506+056 at $z=0.3365$ (IceCube Collaboration et al. 2018a; IceCube Collaboration 2018b; Padovani et al. 2018; Paiano et al. 2018). Other possible associations include 3HSP J095507.9+355101, an extreme blazar with synchrotron peak frequency $^{2} v_{\text {peak }}^{S} \gtrsim 5 \times 10^{17} \mathrm{~Hz}, z=0.557$, and well within the neutrino event error region of IceCube-200107A, that exhibited a very

\footnotetext{
2 From a spectral energy distribution (SED) point of view blazars are divided based on the rest-frame frequency of the low-energy (synchrotron) hump $\left(v_{\text {peak }}^{S}\right)$ into low- (LBL/LSP: $\left.v_{\text {peak }}^{S}<10^{14} \mathrm{~Hz}[<0.41 \mathrm{eV}]\right)$, intermediate-
} 
hard X-ray flare at the time of the IceCube event (Paiano et al. 2020a; Giommi et al. 2020b). The case for some blazars being neutrino sources is mounting (see e.g. Righi, Tavecchio, \& Inoue 2019).

Blazars are a rare type of Active Galactic Nuclei (AGN; see Falomo, Pian \& Treves 2014; Padovani et al. 2017, for reviews) characterised by strong and highly variable radiation across the entire electromagnetic spectrum. This is produced by energetic charged particles moving in a magnetic field within a relativistic jet that is seen at a small angle with respect to the line of sight (Urry \& Padovani 1995; Padovani et al. 2017).

From an optical spectroscopy point of view, blazars have always been divided in two classes, i.e., flat-spectrum radio quasars (FSRQs) and BL Lac objects (henceforth, BLL). FSRQs display strong, quasar-like broad emission lines while BLL sometimes exhibit only absorption features, in some cases show at most weak (a few $\AA$ ) emission lines, and often are totally featureless (Urry \& Padovani 1995). Note that most FSRQs are LBLs, with a small number of them being IBLs.

Several studies reported hints of a correlation between blazars and the arrival direction of astrophysical neutrinos (e.g. Padovani et al. 2016; Lucarelli et al. 2019 and references therein). Very recently some of the authors of this paper have extended the detailed dissection of the region around the IceCube-170922A event related to TXS 0506+056 carried out by Padovani et al. (2018) to all the 70 public IceCube high-energy neutrinos that are well reconstructed (so-called tracks) (Giommi et al. 2020a). This provided a $3.2 \sigma$ $\left(\right.$ post-trial ${ }^{3}$ ) correlation excess of $\gamma$-ray detected IBLs and HBLs with IceCube neutrinos. No excess was found for LBLs. Given that TXS 0506+056, as 3HSP J095507.9+355101, is also a blazar of the IBL/HBL type (Padovani et al. 2019) this result, together with previous findings, consistently points to a growing evidence for a connection between some IceCube neutrinos and IBL and HBL blazars. Moreover, this means that out of the 47 IBLs and HBLs in Table 5 of Giommi et al. $(2020 a) \approx 20$ could be new neutrino sources waiting to be identified (see their Table 6).

To make further progress at this point we need optical spectra, which are required to determine the redshift, and hence the luminosity involved, measure the main properties of the spectral lines, and possibly estimate the mass of the central black hole.

In this paper we present the spectroscopy of a fraction of the $47 \mathrm{ob}-$ jects selected by Giommi et al. (2020a), which together with results taken from the literature cover $\sim 80$ per cent of the sample. The paper is the first of a series of publications arising from the project "The spectra of IceCube Neutrino (SIN) candidate sources" whose purpose is to: (1) determine the nature of the sources, e.g. are they EHBLs like 3HSP J0955 or are they "masquerading BL Lacs"? Padovani et al. (2019), in fact, have shown that, despite appearances, TXS 0506+056 is not a blazar of the BL Lac type but instead a masquerading BL Lac, i.e., intrinsically an FSRQ with hidden broad lines. In other words, its extremely weak emission lines are due to a very bright, Doppler-boosted jet continuum, which is washing out the lines. Are

(IBL/ISP: $10^{14} \mathrm{~Hz}<v_{\text {peak }}^{S}<10^{15} \mathrm{~Hz}[0.41-4.1 \mathrm{eV}$ )], and high-energy (HBL/HSP: $v_{\text {peak }}^{S}>10^{15} \mathrm{~Hz}[>4.1 \mathrm{eV}]$ ) peaked sources respectively (Padovani \& Giommi 1995; Abdo et al. 2010). Extreme blazars are characterized by $v_{\text {peak }}^{S}>2.4 \times 10^{17} \mathrm{~Hz}[>1 \mathrm{keV}]$ (Biteau et al. 2020).

3 A trial correction for the "Look Elsewhere Effect" is needed (e.g., Patrignani $\&$ Particle Data Group 2016). This stems from a simple fact: in the ideal case of 20 completely independent tests, for example, a result more significant than $\sim 2 \sigma$ will be observed simply by chance. The final p-value can in this case be trial-corrected by multiplying it by the number of tests carried out. they extreme blazars as 3HSP J095507.9+355101? (2) model their SEDs using all available multi-wavelength data, including the spectral slopes and the nucleus-to-host ratios (when measured) derived in this paper, starting with the objects with available redshift to determine the physical parameters, which affect the efficiency of neutrino production, and subsequently the expected neutrino emission from each blazar; (3) determine the likelihood of a physical connection between the neutrino and the blazar, as some of us did in Petropoulou et al. (2015) (see also, e.g., Petropoulou et al. 2020 for 3HSP J095507.9+355101), and derive important insight on the physical parameters of the blazar jet, such as its proton content. All of the above will provide fundamental information to study the neutrino - blazar connection.

The present paper is also a continuation of the ongoing systematic study of the properties of Fermi BL Lacs, which some of the present authors have carried out in the last decade (e.g Paiano et al. 2017a,b,c; Landoni, et al. 2018; Paiano et al. 2019a, 2020b). This study has provided measurements of the redshifts and line properties (if detected) of $\gamma$-ray blazars, which are useful to address several related research questions, namely their evolution, their connection with ultra-highenergy CRs, and the EBL and Galactic and extragalactic magnetic fields.

This paper is structured as follows. Section 2 describes the sample we used, while Section 3 deals with the observations and data reduction. Section 4 discusses our methods and results, while Section 5 provides notes on individual sources. Finally, Section 6 summarizes our conclusions.

We use a $\Lambda \mathrm{CDM}$ cosmology with Hubble constant $H_{0}=70 \mathrm{~km}$ $\mathrm{s}^{-1} \mathrm{Mpc}^{-1}$, matter density $\Omega_{\mathrm{m}, 0}=0.3$, and dark energy density $\Omega_{\Lambda, 0}=0.7$.

\section{THE SAMPLE}

Our sample includes all 47 IBLs and HBLs in Table 5 of Giommi et al. (2020a) plus M87. These are all $\gamma$-ray sources and have by selection $\left|b_{\mathrm{II}}\right|>10^{\circ}$ and $v_{\text {peak }}^{S}>10^{14} \mathrm{~Hz}$. These sources are matched to 30 neutrino events out of 70 with reasonably well-defined IceCube positions (i.e., with an area of the error ellipse smaller than that of a circle with radius $r=3^{\circ}$ ). In various cases more than one blazar is within the IceCube error ellipse; the source list is reported in Table 1.

A literature scan shows that for 19 of them a redshift is known (see Table 1) and in three cases it is rather uncertain. Of the remaining 28 objects, we performed spectroscopy and present the spectra for 17. Combining these observations with the literature results, one has a coverage of 36 out of the 47 objects in Giommi et al. (2020a), which corresponds to $\sim 80$ per cent. The 11 objects left are targets of current ongoing observations by our group and will be discussed in a future publication. A journal of the observations of the 17 sources is given in Table 2. Additional spectroscopic observations of observed sources of still unknown redshift are planned with different instrument configuration.

We also observed some targets included in a preliminary version of the Giommi et al. (2020a)'s list. These are still $\gamma$-ray emitting blazars without a redshift determination, which turned out not to fulfil all the final criteria adopted by those authors, especially in regards to the size of the IceCube error ellipse and the $v_{\text {peak }}^{S}$ cut. These seven additional targets are discussed in Appendix A. 


\section{OBSERVATIONS AND DATA REDUCTION}

The optical spectra were collected using two different instruments. For seven sources in the northern hemisphere we used the $10.4 \mathrm{~m}$ Gran Telescopio Canarias (GTC) at the Roque de Los Muchachos (La Palma) with the spectrograph OSIRIS (Cepa et al. 2003). For the other 10 targets the $8 \mathrm{~m}$ Very Large Telescope (VLT) at Paranal equipped with FORS2 (Appenzeller et al. 1998) was utilized. In the former case the instrument was configured with the grism R1000B (slit width of 1.2") that covers the spectral range $4100-7750 \AA$ at a resolution $\mathrm{R} \sim 600$. In the latter case, grism GRIS_300V+10 was used (slit width of 1.3") covering $4700-8600 \AA$ at $R \sim 200$.

Data reduction of the GTC observations was performed adopting standard IRAF procedures (Tody 1986, 1993) for long slit spectroscopy following the same scheme given in Paiano et al. (2017a). For FORS2 data we adopted the reduction pipeline provided by the EsoReflex environment (Freudling et al. 2013) including extraction of $1 \mathrm{D}$ spectra.

In both cases the accuracy of the wavelength calibration based on the scatter of the polinomial fit (pixel vs wavelength) is $\sim 0.1 \AA$ over the whole observed spectral range.

In order to perform an optimal correction of CRs and other artifacts, the observation of each source was divided in at least three individual exposures. This procedure allows us also to check for possible spurious features. The final spectrum results from the combination of all individual exposures.

Spectro-photometric standard stars were secured for each night in order to perform the relative flux calibration. In addition we assessed the absolute flux calibration from the measured magnitude of the source (see Table 3 ) as derived from the acquisition images obtained during the observation. For each acquisition image we measure the observed magnitude of a number of stars with known magnitude from SDSS and/or Pan-STARRS catalogues and derive the calibration constant of the frame. In all cases the accuracy is better than 0.1 magnitude. We note that for about $\sim 50$ per cent of the observed sources we found a difference of $\sim 0.5$ - 1 mag between the present observations and previous epochs (see Section 5 and Table 1 and 3).

Finally all spectra were dereddened applying the extinction law of Cardelli, Clayton \& Mathis (1989) and assuming the value of Galactic extinction E(B-V) derived from the NASA/IPAC Infrared Science Archive ${ }^{4}$ (Schlafly \& Finkbeiner 2011).

\section{RESULTS}

All flux calibrated and dereddened spectra of the 17 neutrino blazar candidates are displayed in Fig. 1. They are also available in the online database ZBLLAC ${ }^{5}$ (Landoni et al. 2020). For each spectrum, we evaluate the signal-to-noise ratio $(\mathrm{S} / \mathrm{N})$ in a number of spectral regions and the averaged value is given in Table 3.

From the spectroscopic point of view, we find that all observed targets but one, 4FGL J0244.7+1316, which has a quasar-like spectrum, can be classified as BLL. Their spectra are characterized by a power-law emission and in some cases also the signature of the host galaxy stellar population is present.

The spectra were carefully inspected in order to identify emission and/or absorption features. When a feature was found, we checked its reliability verifying that it is present in the individual exposures. For 9 out of 17 targets, we are able to detect absorption and/or emission

\footnotetext{
${ }^{4}$ http://irsa.ipac.caltech.edu/applications/DUST/

5 http://web.oapd.inaf.it/zbllac/
}

lines that allow us to determine a firm redshift, which ranges between 0.09 and 1.6 (see Table 3).

For 6 objects the optical spectrum is characteristic of BLL where both the non-thermal component and the host galaxy are clearly visible (Falomo, Pian \& Treves 2014). In Table 5 we report the EW of the main absorption features of the host galaxies. For these targets we have decomposed the observed spectrum into a power law and a template for the host galaxy (Mannucci et al. 2001). The decomposition was obtained from a best fit of the two components with free parameters (nucleus-to-host ratio, spectral slope of the non thermal component). In all above cases the decomposition is a good representation of the observed spectrum (see Figure 3). We find that the ratio (evaluated at $6500 \AA$ ) of these two components, the nucleusto-host ratio (N/H), ranges from 0.3 to 5 (see also Section 5).

It is of interest to search for narrow emission lines as signature of recent star formation and/or nuclear activity in the BLL (Bressan, et al. 2006; Paiano et al. 2017a). We measure the equivalenth width (EW) of these features by integrating the flux of the line above the local linear continuum and estimated the dominant error by assuming slightly different continua (based on the $1 \sigma$ level difference from the assumed continuum). In 5 objects we detected weak and narrow emission lines due to $[\mathrm{O} \mathrm{II}](\mathrm{EW}=0.6-1.0 \AA)$, [O III] $(\mathrm{EW}=0.4$ $1.7 \AA),[\mathrm{N} \mathrm{II}](\mathrm{EW} \sim 1.3 \AA)$ and [S II] and [Ne V]. For 3 sources of known redshift no emission lines of $[\mathrm{O}$ II $]$ and $[\mathrm{O}$ III] are detected and we set an upper limit of the line luminosity based on the estimated minimum Equivalent Width (EW) at the expected wavelength of the line. Three sources exhibit broad emission lines due to Mg II, C III] and C II]. The properties of these emission lines are summarized in Table 4. For 4FGL J2227.9+0036 only absorption systems due to an intervening medium are observed and therefore only a lower limit to the redshift can be derived.

Finally for the remaining seven sources the optical spectrum appears featureless and well described by a power law continuum. For these objects, we evaluate the minimum detectable EW from which a lower limit of the redshift $(0.25-0.7$, see also Table 3$)$ is derived following the procedure described in Paiano et al. (2017a). Briefly it is assumed that the optical spectrum is the superposition of a non-thermal component described by a power law $\left(\mathrm{F}_{\lambda} \sim \lambda^{\alpha}\right)$ and the starlight component of an elliptical host galaxy of $\mathrm{M}(\mathrm{R})=-22.9$ (Sbarufatti et al. 2005). Under the above assumption, the visibility of the stellar features depend on the nucleus-to-host ratio and therefore from the observed magnitude of the source and the minimum EW of the spectrum a lower limit to the redshift can be estimated. Since the distribution of the absolute magnitude of the host galaxies of BL Lacs has a width of $\sim 1$ magnitude (see Sbarufatti et al. (2005)) the redshift lower limits given in Table represent the most probable value. If the host galaxy of a source is 0.5 mag lower or higher than the assumed average value also the redshift limit will be greater or smaller by $\sim 0.05-0.1$ (depending on the redshift) respectively.

\section{NOTES ON INDIVIDUAL SOURCES}

4FGL J0224.2+1616: We obtained a high quality S/N ( 70) spectrum of the object $(\mathrm{g}=19.8)$. The spectrum is characterized by a featureless continuum that is described by a power law $(\alpha \sim-0.80)$ taking into account a significant reddening $(\mathrm{E}(\mathrm{B}-\mathrm{V})=0.20)$. On the basis of the minimum EW $(\sim 0.4 \AA)$ a lower limit of the redshift $\mathrm{z}>0.5$ can be set due to the lack of absorption by the starlight of the host galaxy. Under the above condition, in the spectral range from $5500-6500 \AA$ we can set an upper limit to the flux for possible narrow emission lines $\lesssim 5 \times 10^{-17} \mathrm{erg} \mathrm{cm}^{-2} \mathrm{~s}^{-1}$. 
4FGL J0239.5+1326: We secured a spectrum $(\mathrm{S} / \mathrm{N} \sim 45)$ of the object (with a magnitude $\mathrm{g}=20.6$ ), characterized by a featureless continuum and described by a power law $(\alpha \sim-0.55)$ considering the reddening with $\mathrm{E}(\mathrm{B}-\mathrm{V})=0.10$. The upper limit on the $\mathrm{EW}$ for the lines is $0.5 \AA$ and from the non detection of absorption lines from the host galaxy, we set a lower limit of the redshift of $z>0.7$. This condition implies a line flux $\lesssim 2.0 \times 10^{-17} \mathrm{erg} \mathrm{cm}^{-2} \mathrm{~s}^{-1}$ for possible narrow emission lines at the spectral range 5500 - $6500 \AA$.

4FGL J0244.7+1316: Our optical spectrum exhibits a prominent $(E W \sim 124 \AA, F W H M \sim 74 \AA)$ emission line at $5557 \AA$ identified as $\mathrm{Mg}$ II 2800 at $\mathrm{z}=0.9846$. Two additional narrow emission features attributed to $[\mathrm{Ne} \mathrm{V}]$ and [O II] are clearly detected (see Table 4 and Fig. 2) at the same redshift. The object has a clear quasar-like appearance.

4FGL J0344.4+3432: Our spectrum of the target $(\mathrm{g}=19.7)$ is featureless and described by a power law $(\alpha \sim-0.90)$ with a minimum detectable EW 0.9 $\AA$. This allows us to set a lower limit to the redshft $\mathrm{z}>0.25$ considering an elliptical host galaxy for the BLL. Under the above assumption, we set an upper limit line flux of $\lesssim 1.3 \times 10^{-16} \mathrm{erg} \mathrm{cm}^{-2} \mathrm{~s}^{-1}$ for possible narrow emission lines in the spectral range 5500 - $6500 \AA$.

4FGL J0525.6-2008: The optical spectrum shows the typical absorption features (G-band, $\mathrm{H}_{\beta}, \mathrm{Mg} \mathrm{I}, \mathrm{Ca}+\mathrm{Fe}, \mathrm{Na} \mathrm{I}$ ) of the old stellar population characteristic of elliptical galaxies at $\mathrm{z}=0.0913$. Indeed from the images (see e.g. the Pan-STARRS images), the target appears as an elliptical galaxy of $g=17.1$ (from aperture photometry of radius 2.7 arcsec). In addition to the absorption features, we also detect weak emission lines of [O III] 5007 (see Fig. 2), [N II] 6548,6584, and [S II] 6716-6731 (see Table 4) at the same redshift. From the spectral decomposition (see Fig. 3), we derive a nucleus-to-host ratio $\mathrm{N} / \mathrm{H} \sim 4.5$ and $\alpha=-1.0$ for the nuclear power law component.

4FGL J0649.5-3139: From the optical spectrum obtained by Peña-Herazo et al. (2017) (and reported in the ZBLLAC database), an absorption feature at $\sim 4400 \AA$ was found. The identification with $\mathrm{Mg}$ II from intervening absorption sets a lower limit to the redshift $\mathrm{z} \geq 0.563$. We obtain a better quality ( $\mathrm{S} / \mathrm{N} \sim 210$ ) optical spectrum to search for possible emission lines in order to determine a firm redshift. Our spectrum is featureless and exhibits a continuum power law $(\alpha \sim-1.15)$. No emission lines are present with EW $>0.20 \AA$ corresponding to an upper limit of line flux of $\lesssim 9 \times 10^{-18} \mathrm{erg} \mathrm{cm}^{-2} \mathrm{~s}^{-1}$ for possible narrow emission lines in the spectral range 5500 - $6500 \AA$. We cannot confirm the absorption feature at $\sim 4400 \AA$ because it is outside our spectral range.

4FGL J0854.0+2753: A noisy SDSS spectrum suggests a redshift $\mathrm{z}=0.4937$. We obtained a moderate $\mathrm{S} / \mathrm{N}$ spectrum for which we clearly detect the absorption features of Ca II (at 5873, $5925 \AA$ ) and G-band (at $6426 \AA$ ), obtaining $\mathrm{z}=0.4930$.

4FGL J1040.5+0617: The tentative redshift $\mathrm{z}=0.735$ was proposed by Maselli et al. (2015) on the basis of a single weak emission line from a SDSS spectrum. We obtained a high quality $\mathrm{S} / \mathrm{N} \sim 120$ spectrum that allows us to clearly detect a broad emission line $(\mathrm{EW}=4.69 \pm 0.5 \AA$ ) attributed to $\mathrm{Mg}$ II 2800 at $4874 \AA$ and a narrow line due to [O II] 3727 at $6468 \AA$ (see the close up in Fig. 2). The redshift is $\mathrm{z}=0.740$.

4FGL J1043.6+0654: We obtain a high quality $\mathrm{S} / \mathrm{N}(\sim 150)$ of the source $(\mathrm{g}=19.4)$. The spectrum is characterized by a featureless continuum and well described by a power law with $\alpha=-1.15$. No emission or absorption lines are detected down to $\mathrm{EW} \sim 0.20 \AA$ that yields a lower limit of the redshift of $\mathrm{z}>0.7$ and a line flux $\lesssim 1.2 \times 10^{-17} \mathrm{erg} \mathrm{cm}^{-2} \mathrm{~s}^{-1}$ for possible narrow emission lines at the spectral range $5500-6500 \AA$.

4FGL J1258.7-0452: In our spectrum the Ca II absorption doublet is apparent at 5576, $5627 \AA$ (see Fig. 2) and, together with the G-band absorption line at $6103 \AA$, yields $\mathrm{z}=0.4179$. No emission lines are present with $\mathrm{EW}>0.3 \AA$. This corresponds to an [O II] and an [O III] line luminosity $\lesssim 3.0 \times 10^{40} \mathrm{erg} \mathrm{s}^{-1}$ and $\lesssim 2 \times 10^{40} \mathrm{erg} \mathrm{s}^{-1}$ respectively. The decomposition of the spectrum (see Fig. 3) provides a $\mathrm{N} / \mathrm{H} \sim 5$, and $\alpha=-1.6$ for the nuclear power law component.

4FGL J1300.0+1753: A noisy SDSS spectrum does not display any significant features. Our spectrum $(\mathrm{S} / \mathrm{N} \sim 40)$ is dominated by a power law continuum emission with $\alpha \sim-1.30$. No features are detected down to $\mathrm{EW}=0.7 \AA$, corresponding to a lower limit of $\mathrm{z}>0.6$ based on the non-detection of the absorption features from the starlight of the elliptical host galaxy. We can set an upper limit line flux of $\sim 2.5 \times 10^{-17} \mathrm{erg} \mathrm{cm}^{-2}$ for narrow emission lines in the spectral range $5500-6500 \AA$.

4FGL J1359.1-1152: Our spectrum is characterized by the typical absorption lines (Ca II, G-band, $\mathrm{H}_{\beta} \mathrm{Mg} \mathrm{I}, \mathrm{Ca}+\mathrm{Fe}$, $\mathrm{Na} \mathrm{I}$ and $\mathrm{H}_{\alpha}$ ) due to old stellar population of the host galaxy at the redshift $\mathrm{z}=0.242$. No emission line are detected down to $\mathrm{EW}=0.6 \AA$. This corresponds to an [OIII] line luminosity $\lesssim 2.1 \times 10^{40} \mathrm{erg} \mathrm{s}^{-1}$. From the decomposition into template galaxy and a non-thermal emission (see Fig. 3), we find a N/H 0.5, and $\alpha=-0.8$ for the nuclear power law component.

4FGL J1440.0-2343: The spectrum is characterized by a nonthermal continuum with the signature of $\mathrm{Ca}$ II doublet and G-band absorption features (see Fig. 2) from the host galaxy at $\mathrm{z}=0.309$. In addition we detect emission lines at $4878 \AA$ ( $\mathrm{EW}=1.0 \AA$ ) and $6553 \AA$ (EW = $0.4 \AA)$, that we identify with [O II] and [O III] at the same redshift. The host galaxy is well detected in the decomposition (see Fig. 3) where $\mathrm{N} / \mathrm{H}=2.5$ and the power law index $\alpha \sim-1$ of the nuclear component are estimated.

4FGL J1447.0-2657: The spectrum shows the absorption lines due to $\mathrm{Ca}$ II, G-band, and $\mathrm{H}_{\beta}$ attributed to the stellar population of the host galaxy at $\mathrm{z}=0.3315$. No emission lines are detected in the spectrum. The upper limit on the EW is $\sim 0.5 \AA$ that corresponds to an [OII] and [OIII] line flux of $\lesssim 7.8$ and $6.5 \times 10^{-17} \mathrm{erg} \mathrm{cm}^{-2} \mathrm{~s}^{-1}$ and a line luminosity upper limit $\lesssim 2.8$ and $2.5 \times 10^{40} \mathrm{erg} \mathrm{s}^{-1}$. From the decomposition (see Fig. 3) the spectrum is characterized by a non-thermal power law continuum with $\alpha \sim-1.3$ and we find a $\mathrm{N} / \mathrm{H}=1.5$.

4FGL J2223.3+0102: There are two spectra secured by the SDSS survey. In both cases no convincing absorption or emission features are apparent. We obtained a higher quality spectrum of the target $(\mathrm{g} \sim 19.3)$. The continuum is well described by a power law index of $\alpha=-0.80$. In spite of the much higher $\mathrm{S} / \mathrm{N}(\sim 150)$ no spectral features are detected with $\mathrm{EW}>0.15 \AA$ and this implies an upper limit to the line flux $\lesssim 1.5 \times 10^{-17} \mathrm{erg} \mathrm{cm}^{-2} \mathrm{~s}^{-1}$ for possible narrow emission lines in the spectral range $5500-6500 \AA$ Ar. From the non detection of the absorption lines of the host galaxy, we set a redshift lower limit of $\mathrm{z}>0.7$.

4FGL J2227.9+0036: The spectrum is dominated by a nonthermal continuum well described by a power law $(\alpha=-0.70)$. We detect an absorption doublet at 5853, $5868 \AA$ and $\mathrm{EW}=1.30,0.80 \AA$ (see Fig. 1 and Fig. 2) that corresponds to a Mg II intervening absorption system at $\mathrm{z}=1.0935$. This feature is also present in a medium-quality SDSS spectrum, but it was not correctly identified because such features are not searched for by the SDSS pipeline. Two addition absorption lines are found at $4988 \AA(\mathrm{EW}=0.70 \AA$ ) and $5443 \AA$ (EW $=0.30 \AA)$ corresponding to Fe II $(2382,2600)$ at the same redshift. Therefore the target is at $\mathrm{z}>1.0935$. No emission lines are detected in the spectrum. Taking into account the redshift lower limit, the $[\mathrm{O} \mathrm{II}]$ emission lines can be located at $\gtrsim 7800 \AA$ 
where the minimum EW is $0.5 \AA$. This corresponds to a line flux $\lesssim 3.5 \times 10^{-17} \mathrm{erg} \mathrm{cm}^{-2} \mathrm{~s}^{-1}$.

4FGL J2326.2+0113: The SDSS spectrum shows some emission lines, which suggest a redshift $\mathrm{z}=1.59$. We obtained a better $\mathrm{S} / \mathrm{N}$ $(\sim 120)$ spectrum that is characterized by a power law continuum $(\alpha$ $\sim-0.25$ ). We clearly detect emission lines of C III], C II], and Mg II at $\mathrm{z}=1.597$ (see Fig. 1 and Tab. 4). In addition we also detect an $\mathrm{Mg}$ II intervening absorption system at $\mathrm{z}=1.084$.

\section{SUMMARY AND FUTURE PERSPECTIVES}

We presented optical spectroscopy of 17 extragalactic sources that are candidate for being the astronomical counterparts of neutrino events. Apart from one target, which has a quasar-like spectrum (4FGL J0244.7+1316), all of the remaining objects have a spectral shape that is well consistent with a BLL classification. We clearly detected absorption and/or emission features in the spectra of 10 targets. For 9 of them we are able to determine a firm redshift $(0.09<\mathrm{z}<1.6)$ from absorption lines due to the old stellar population of their host galaxies and in 5 cases also from the emission lines. For one source (4FGL J2227.9+0036) we set a spectroscopic lower limit to the redshift, based on the detection of intervening absorption systems attributed to Mg II and Fe II. Five sources show in their optical spectra the clear modulation due to the flux from the host galaxy (see Figure 3$)$. The closest object $(\mathrm{z}=0.0913)$ in our sample is 4FGLJ0525.6-2008 and its optical spectrum is fully dominated by the host galaxy. Despite the good quality of the spectra in seven objects we do not detect any spectral feature as is expected for this class of sources. Nevertheless for these we can estimate a lower limit to the redshift from the absence of the absorption lines of their host galaxy. Only for five sources the spectrum exhibits emission features (see Table 4). These are forbidden lines attributed to [O II], [O III] and $[\mathrm{N} \mathrm{II}]$ of luminosity $(1-4) \times 10^{41}, \sim 3 \times 10^{40}$, and $\sim 2 \times 10^{41} \mathrm{erg} \mathrm{s}^{-1}$ respectively. Moreover, semi-forbidden lines of C III] and C II] are revealed in the high redshift $(\mathrm{z}=1.595)$ BLL 4FGL J2326.2+0113 and a broad emission line of Mg II $2800 \AA$ is found in the spectra of two targets. Overall the spectral properties of the objects in this study are similar to those of a sample of 55 hard $\gamma$-ray BLL investigated by Paiano et al. (2020b) both as regards the continuum shape and redshift range of the objects. In both samples, when weak [O III] 5007 emission line is detected, their luminosity is comparable (in the range $\left.L_{[O I I I]}=(2-8) \times 10^{40} \mathrm{erg} \mathrm{s}^{-1}\right)$. These issues will be fully discussed in the second paper of this series.

We will be using the results of this paper to carry out a detailed investigation of the nature of these blazars. In particular, following Padovani et al. (2019) and based on the continuum and line powers, and the overall SEDs, we will be assessing if any of these blazars can be classified as "masquerading", as is the case for TXS 0506+056, or extreme BLL as 3HSP J095507.9+355101. Moreover to study in depth the neutrino - blazar connection with a statistically meaningful sample, a model of their photon and neutrino SEDs using a leptohadronic code (e.g. Petropoulou et al. 2020) will be explored. 
Table 1. The sample of 47 neutrino candidate blazars. In boldface are the targets considered in this paper.

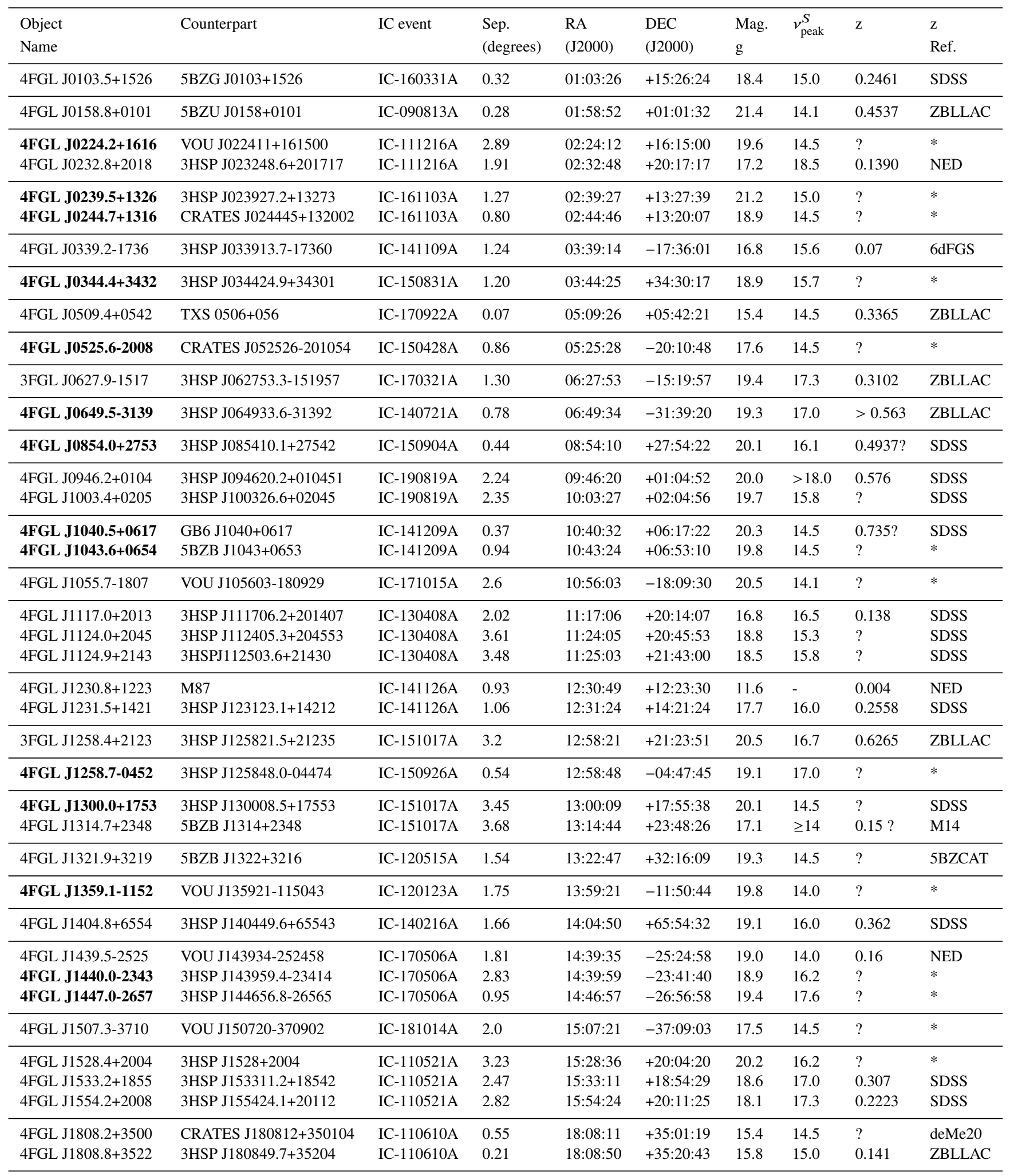

Notes. Column 1: Fermi name of the target in the 4FGL (or 3FGL in two cases) catalogue; Column 2: Counterpart name of the $\gamma$-ray source; Column 3: IceCube track; Column 4: Angular separation (degrees) between the target and the centroid of the IceCube track; Column 5 - 6: Right ascension and declination of the optical counterpart; Column 7: g magnitude from SDSS survey and PANSTARRs; Column 8: Frequency of the synchrotron peak $\left(v_{\text {peak }}^{\text {syn }}\right.$ ); Column 9: Redshift from the literature; Column 10: Reference for the redshift (SDSS: SDSS Data Release 16 (DR16): Ahumada et al. (2020); ZBLLAC: Database of BL Lac objects spectra - http://www.oapd.inaf.it/zbllac/; NED: NASA/IPAC Extragalactic Database; 6dFGS: 6dF Galaxy Survey Database - Final Data Release (DR3): Jones et al. (2009); M14: Massaro et al. 2014; deMe20: de Menezes et al. 2020). VOU: Giommi et al. (2020a); 5BZCAT: Massaro et al. (2015); an asterisk means that no redshift or optical spectra are found in the literature. 
Table 1. The sample of 47 neutrino candidate blazars - continued

\begin{tabular}{|c|c|c|c|c|c|c|c|c|c|}
\hline $\begin{array}{l}\text { Object } \\
\text { Name }\end{array}$ & Counterpart & IC event & $\begin{array}{l}\text { Sep. } \\
\text { (degrees) }\end{array}$ & $\begin{array}{l}\text { RA } \\
(\mathrm{J} 2000)\end{array}$ & $\begin{array}{l}\text { DEC } \\
(\mathrm{J} 2000)\end{array}$ & $\begin{array}{l}\text { Mag. } \\
\text { g }\end{array}$ & $v_{\text {peak }}^{S}$ & $\mathrm{z}$ & $\begin{array}{l}\text { z } \\
\text { Ref. }\end{array}$ \\
\hline 4FGL J2030.5+2235 & 3HSP J203031.6+22343 & IC-100710A & 1.31 & $20: 30: 32$ & $+22: 34: 39$ & 20.3 & 16.2 & $?$ & $*$ \\
\hline 4FGL J2030.9+1935 & 3HSP J203057.1+19361 & IC-100710A & 1.82 & $20: 30: 57$ & $+19: 36: 12$ & 18.6 & 15.8 & $?$ & $*$ \\
\hline 4FGL J2133.1+2529c & 3 HSPJ213314.3+25285 & IC-150714A & 2.18 & $21: 33: 14$ & $+25: 28: 59$ & 18.8 & 15.2 & 0.294 & 5BZCAT \\
\hline 4FGL J2223.3+0102 & 3HSP J222329.5+01022 & IC-140114A & 1.33 & $22: 23: 30$ & $+01: 02: 26$ & 18.6 & 15.5 & $?$ & SDSS \\
\hline 4FGL J2227.9+0036 & 5BZB J2227+0037 & IC-140114A & 0.64 & $22: 27: 58$ & $+00: 37: 06$ & 18.4 & 14.5 & $?$ & SDSS \\
\hline 4FGL J2326.2+0113 & CRATES J232625+011147 & IC-160510A & 1.31 & $23: 26: 26$ & $+01: 12: 09$ & 20.5 & 14.0 & $?$ & SDSS \\
\hline 4FGL J2350.6-3005 & 3HSPJ235034.3-30060 & IC-190104A & 3.32 & $23: 50: 34$ & $-30: 06: 03$ & 18.1 & 15.7 & 0.2328 & 6dFGS \\
\hline 4FGL J2351.4-2818 & IC 5362 & IC-190104A & 1.07 & $23: 51: 36$ & $-28: 21: 53$ & 16.3 & 14.5 & 0.0276 & NED \\
\hline 4FGL J2358.1-2853 & CRATES J235815-285341 & IC-190104A & 2.47 & $23: 58: 17$ & $-28: 53: 34$ & 19.5 & 14.0 & $?$ & $*$ \\
\hline
\end{tabular}

Table 2. The sample of 17 neutrino candidate blazars and journal of the observations.

\begin{tabular}{llllllll}
\hline Object Name & $\mathrm{E}(\mathrm{B}-\mathrm{V})$ & Telescope & Instrument & $\begin{array}{l}\text { Date } \\
(\mathrm{s})\end{array}$ & $\begin{array}{l}\mathrm{t}_{\text {Exp }} \\
(")\end{array}$ & seeing & Air Mass \\
\hline 4FGL J0224.2+1616 & 0.20 & GTC & OSIRIS & 22 December 2019 & 7200 & 1.7 & 1.30 \\
4FGL J0239.5+1326 & 0.10 & GTC & OSIRIS & 05 October 2019 & 7200 & 1.8 & 1.05 \\
4FGL J0244.7+1316 & 0.09 & GTC & OSIRIS & 09 October 2019 & 4500 & 0.7 & 1.04 \\
4FGL J0344.4+3432 & 0.27 & GTC & OSIRIS & 10 October 2019 & 3000 & 0.7 & 1.47 \\
4FGL J0525.6-2008 & 0.04 & VLT & FORS2 & 23 October 2019 & 2400 & 0.8 & 1.06 \\
4FGL J0649.5-3139 & 0.10 & VLT & FORS2 & 23 October 2019 & 5400 & 1.0 & 1.07 \\
4FGL J0854.0+2753 & 0.03 & GTC & OSIRIS & 21 October 2019 & 6600 & 1.4 & 1.25 \\
4FGL J1040.5+0617 & 0.02 & GTC & OSIRIS & 15 March 2019 & 7200 & 1.6 & 1.22 \\
4FGL J1043.6+0654 & 0.03 & VLT & FORS2 & 26 December 2019 & 5400 & 0.5 & 1.35 \\
4FGL J1258.7-0452 & 0.02 & VLT & FORS2 & 18 February 2020 & 5400 & 0.5 & 1.15 \\
4FGL J1300.0+1753 & 0.03 & GTC & OSIRIS & 02 April 2019 & 9000 & 1.7 & 1.31 \\
4FGL J1359.1-1152 & 0.08 & VLT & FORS2 & 28 February 2020 & 2700 & 2.0 & 1.14 \\
4FGL J1440.0-2343 & 0.10 & VLT & FORS2 & 24 March 2020 & 2700 & 1.9 & 1.08 \\
4FGL J1447.0-2657 & 0.11 & VLT & FORS2 & 24 March 2020 & 5400 & 1.3 & 1.00 \\
4FGL J2223.3+0102 & 0.06 & VLT & FORS2 & 04 October 2019 & 5400 & 0.9 & 1.14 \\
4FGL J2227.9+0036 & 0.05 & VLT & FORS2 & 03 October 2019 & 2700 & 0.8 & 1.35 \\
4FGL J2326.2+0113 & 0.03 & VLT & FORS2 & 03 October 2019 & 5400 & 0.7 & 1.17 \\
\hline
\end{tabular}

Notes. Column 1: Name of the target ; Column 2: E(B-V) taken from the NASA/IPAC Infrared Science Archive

(https://irsa.ipac.caltech.edu/applications/DUST/); Column 3: Telescope used for the observation; Column 4: Date of the observation; Column 5: Total integration time (sec); Column 6: Average seeing during the observation ( $\operatorname{arcsec})$, Column 6: Air mass during the observation. 
Table 3. Properties of the optical spectra of the 17 neutrino candidate blazars studied in this work.

\begin{tabular}{llcllll}
\hline Object Name & $\mathrm{g}$ & $\mathrm{S} / \mathrm{N}$ & $\begin{array}{l}\mathrm{EW}_{\text {min }} \\
(\AA)\end{array}$ & $\mathrm{z}$ & Line type & $\alpha$ \\
\hline 4FGL J0224.2+1616 & $19.8 \pm 0.1$ & 70 & 0.40 & $>0.5$ & $\mathrm{~h}$ & $0.80 \pm 0.2$ \\
4FGL J0239.5+1326 & $20.6 \pm 0.2$ & 45 & 0.50 & $>0.7$ & $\mathrm{~h}$ & $0.55 \pm 0.15$ \\
4FGL J0244.7+1316 & $18.9 \pm 0.1$ & 110 & - & $0.9846 \pm 0.0005$ & $\mathrm{e}$ & $1.0 \pm 0.2$ \\
4FGL J0344.4+3432 & $19.7 \pm 0.2$ & 45 & 0.55 & $>0.25$ & $\mathrm{~h}$ & $0.90 \pm 0.2$ \\
4FGL J0525.6-2008 & $17.6 \pm 0.1$ & 200 & - & $0.0913 \pm 0.0005$ & $\mathrm{e}, \mathrm{g}$ & $1.00 \pm 0.2$ \\
4FGL J0649.5-3139 & $20.3 \pm 0.2$ & 210 & 0.20 & $>0.7$ & $\mathrm{~h}$ & $1.15 \pm 0.2$ \\
4FGL J0854.0+2753 & $19.9 \pm 0.1$ & 15 & - & $0.4930 \pm 0.0005$ & $\mathrm{~g}$ & $0.80 \pm 0.2$ \\
4FGL J1040.5+0617 & $19.4 \pm 0.1$ & 120 & - & $0.740 \pm 0.001$ & $\mathrm{e}$ & $0.75 \pm 0.15$ \\
4FGL J1043.6+0654 & $19.7 \pm 0.1$ & 150 & 0.20 & $>0.7$ & $\mathrm{~h}$ & $1.15 \pm 0.2$ \\
4FGL J1258.7-0452 & $18.8 \pm 0.1$ & 170 & - & $0.4179 \pm 0.0006$ & $\mathrm{~g}$ & $1.6 \pm 0.3$ \\
4FGL J1300.0+1753 & $20.1 \pm 0.2$ & 40 & 0.7 & $>0.6$ & $\mathrm{~h}$ & $1.3 \pm 0.3$ \\
4FGL J1359.1-1152 & $19.4 \pm 0.1$ & 65 & - & $0.242 \pm 0.001$ & $\mathrm{~g}$ & $0.80 \pm 0.1$ \\
4FGL J1440.0-2343 & $18.6 \pm 0.1$ & 90 & - & $0.309 \pm 0.001$ & $\mathrm{e}, \mathrm{g}$ & $1.0 \pm 0.2$ \\
4FGL J1447.0-2657 & $19.4 \pm 0.1$ & 80 & - & $0.3315 \pm 0.0004$ & $\mathrm{~g}$ & $1.30 \pm 0.2$ \\
4FGL J2223.3+0102 & $19.3 \pm 0.1$ & 150 & 0.15 & $>0.7$ & $\mathrm{~h}$ & $0.8 \pm 0.10$ \\
4FGL J2227.9+0036 & $19.3 \pm 0.1$ & 105 & - & $>1.0935$ & $\mathrm{i}$ & $0.7 \pm 0.10$ \\
4FGL J2326.2+0113 & $19.9 \pm 0.2$ & 120 & - & $1.595 \pm 0.001$ & $\mathrm{e}, \mathrm{i}$ & $0.25 \pm 0.05$ \\
\hline
\end{tabular}

Notes. Column 1: Name of the target; Column 2: Magnitude (g) measured from the acquisition image; Column 3: Median S/N of the spectrum; Column 4: Minimum equivalent width $\left(\mathrm{EW}_{\text {min }}\right)$ derived in the 5500 - $6500 \AA$ A range (the measure is provided only in case of featureless spectrum); Column 5: Redshift. The error is evaluated as combination of the uncertanity of the centroid of the features with the overall accuracy of the wavelength calibration; Column 6: Type of detected line to estimate the redshift: $e=$ emission line, $g=$ galaxy absorption line, $i=$ intervening absorption assuming Mg II $2800 \AA$ identification, $h=$ lower limit derived on the lack of detection of host galaxy absorption lines assuming a BLL elliptical host galaxy with $M(R)=-22.9$. Since the distribution of BLL host galaxies has a dispersion $(1 \sigma)$ of $\sim 0.5 \mathrm{mag}$, these limits may be change by 0.05-0.1 depending on the redshift limit (see details in Paiano et al. 2017a). Column-7: Spectral index $\alpha$ of the continuum described by a power law $\mathrm{F}_{\lambda} \sim \lambda^{-\alpha}$

Table 4. Properties of the emission lines.

\begin{tabular}{|c|c|c|c|c|c|}
\hline Object Name & $\mathrm{z}$ & $\begin{array}{l}\lambda \\
(\AA)\end{array}$ & $\begin{array}{l}\text { EW } \\
(\AA)\end{array}$ & Line ID & $\begin{array}{l}L \text { (line) } \\
\left(\mathrm{erg} \mathrm{s}^{-1}\right)\end{array}$ \\
\hline \multirow[t]{3}{*}{ 4FGL J0244.7+1316 } & 0.9846 & 5557 & $124 \pm 3.0$ & Mg II 2800 & $1.5 \times 10^{44}$ \\
\hline & & 6795 & $0.65 \pm 0.2$ & [Ne V] 3426 & $3.8 \times 10^{41}$ \\
\hline & & 7396 & $0.90 \pm 0.2$ & [O II] 3727 & $4.2 \times 10^{41}$ \\
\hline \multirow[t]{3}{*}{ 4FGL J0525.6-2008 } & 0.0913 & 5464 & $1.70 \pm 0.3$ & [O III] 5007 & $2.2 \times 10^{40}$ \\
\hline & & 7184 & $1.35 \pm 0.3$ & [N II] 6584 & $1.9 \times 10^{40}$ \\
\hline & & 7340 & $1.20 \pm 0.2$ & [SII] 6716-6731 & $1.6 \times 10^{40}$ \\
\hline \multirow[t]{2}{*}{ 4FGL J1040.5+0617 } & 0.740 & 4874 & $4.69 \pm 0.5$ & Mg II 2800 & $1.1 \times 10^{42}$ \\
\hline & & 6468 & $0.58 \pm 0.2$ & [O II ] 3727 & $1.1 \times 10^{41}$ \\
\hline \multirow[t]{2}{*}{ 4FGL J1258.7-0452 } & 0.4179 & 5284 & $<0.3$ & [O II] 3727 & $\lesssim 3.0 \times 10^{40}$ \\
\hline & & 7099 & $<0.3$ & [O III] 5007 & $\lesssim 2.0 \times 10^{40}$ \\
\hline 4FGL J1359.1-1152 & 0.242 & 6218 & $<0.6$ & [O III] 5007 & $\lesssim 2.1 \times 10^{40}$ \\
\hline \multirow[t]{2}{*}{ 4FGL J1440.0-2343 } & 0.309 & 4878 & $0.98 \pm 0.3$ & [O II] 3727 & $7.9 \times 10^{40}$ \\
\hline & & 6553 & $0.36 \pm 0.2$ & [O III] 5007 & $2.8 \times 10^{40}$ \\
\hline \multirow[t]{2}{*}{ 4FGL J1447.0-2657 } & 0.3315 & 4962 & $<0.6$ & [O II] 3727 & $\lesssim 2.8 \times 10^{40}$ \\
\hline & & 6667 & $<0.5$ & [O III] 5007 & $\lesssim 2.5 \times 10^{40}$ \\
\hline \multirow[t]{3}{*}{ 4FGL J2326.2+0113 } & 1.595 & 4953 & $2.67 \pm 0.6$ & C III] 1909 & $2.6 \times 10^{42}$ \\
\hline & & 6041 & $0.82 \pm 0.3$ & C II] 2326 & $7.4 \times 10^{41}$ \\
\hline & & 7272 & $0.81 \pm 0.3$ & Mg II 2800 & $7.1 \times 10^{41}$ \\
\hline
\end{tabular}

Notes. Column 1: Name of the target; Column 2: Redshift; Column 3: Barycenter of the detected line; Column 4: Measured equivalent width of the line; Column 5: Line identification; Column 6: Line luminosity. 
Table 5. Equivalent width of the main host galaxy absorption lines.

\begin{tabular}{llllll}
\hline Object Name & Redshift & $\begin{array}{l}\text { Ca II } \\
(\AA)\end{array}$ & $\begin{array}{l}\text { G-band } \\
(\AA)\end{array}$ & $\begin{array}{l}\text { Mg I 5175 } \\
(\AA)\end{array}$ & $\begin{array}{l}\text { Na I 5892 } \\
(\AA)\end{array}$ \\
\hline 4FGL J0525.6-2008 & 0.0913 & $*$ & $8.0 \pm 1$ & $25 \pm 5$ & $6 \pm 1$ \\
4FGL J0854.0+2753 & 0.4930 & $18 \pm 3$ & $6 \pm 2$ & $*$ & $*$ \\
4FGL J1258.7-045 & 0.4179 & $2.5 \pm 0.5$ & $1 \pm 0.3$ & $*$ & $*$ \\
4FGL J1359.1-115 & 0.242 & $15 \pm 1$ & $6 \pm 0.5$ & $9 \pm 1$ & $*$ \\
4FGL J1440.0-234 & 0.309 & $3.5 \pm 0.5$ & $1.3 \pm 0.2$ & $3 \pm 0.7$ & $*$ \\
4FGL J1447.0-265 & 0.3315 & $6.5 \pm 0.7$ & $2.5 \pm 0.8$ & $18 \pm 3$ & $*$ \\
\hline
\end{tabular}

Notes. Column 1: Name of the target; Column 2: Redshift; Column 3: EW of the doublet CaII 3934, $3968 \AA$; Column 4: EW of G-band 4304 Å; Column 5: EW of Mg I $5175 \AA$ Alend; Column 6: EW of Na I $5892 \AA$. EW measurements and errors follow the procedure outlined in Section 4 for the emission lines. 


\section{ACKNOWLEDGMENTS}

We acknowledge helpful discussions and suggestions by Maria Petropoulou and Foteini Oikonomou. This work is based on observations collected at the European Southern Observatory under ESO programme 0104.B-0032(A) and Gran Telescopio Canarias under the programme GTC24-19B. Funding for the Sloan Digital Sky Survey IV has been provided by the Alfred P. Sloan Foundation, the U.S. Department of Energy Office of Science, and the Participating Institutions. SDSS-IV acknowledges support and resources from the Center for High Performance Computing at the University of Utah. The SDSS website is www.sdss.org. SDSSIV is managed by the Astrophysical Research Consortium for the Participating Institutions of the SDSS Collaboration including the Brazilian Participation Group, the Carnegie Institution for Science, Carnegie Mellon University, Center for Astrophysics | Harvard \& Smithsonian, the Chilean Participation Group, the French Participation Group, Instituto de Astrofísica de Canarias, The Johns Hopkins University, Kavli Institute for the Physics and Mathematics of the Universe (IPMU) / University of Tokyo, the Korean Participation Group, Lawrence Berkeley National Laboratory, Leibniz Institut für Astrophysik Potsdam (AIP), Max-Planck-Institut für Astronomie (MPIA Heidelberg), Max-Planck-Institut für Astrophysik (MPA Garching), Max-Planck-Institut für Extraterrestrische Physik (MPE), National Astronomical Observatories of China, New Mexico State University, New York University, University of Notre Dame, Observatário Nacional / MCTI, The Ohio State University, Pennsylvania State University, Shanghai Astronomical Observatory, United Kingdom Participation Group, Universidad Nacional Autónoma de México, University of Arizona, University of Colorado Boulder, University of Oxford, University of Portsmouth, University of Utah, University of Virginia, University of Washington, University of Wisconsin, Vanderbilt University, and Yale University.

\section{DATA AVAILABILITY}

The flux-calibrated and dereddened spectra are available in our online data base ZBLLAC ${ }^{6}$.

\section{REFERENCES}

Aartsen M. G., Ackermann M., Adams J., Aguilar J. A., Ahlers M., Ahrens M., Alispach C., et al., 2020, PhRvL, 124, 051103

Abdo A. A., Ackermann M., Ajello M., et al., 2010, ApJ, 716, 30

Ahumada R., Prieto C. A., Almeida A., Anders F., Anderson S. F., Andrews B. H., Anguiano B., et al., 2020, ApJS, 249, 3

Appenzeller I., Fricke K., Fürtig W., Gässler W., Häfner R., Harke R., Hess H.-J., et al., 1998, ESO Messenger, 94, 1

Aublin J., 2019, ICRC, 36, 840

Biteau J., Prandini E., Costamante L., Lemoine M., Padovani P., Pueschel E., Resconi E., et al., 2020, NatAs, 4, 124

Bressan A., Falomo R., Valdés J. R., Rampazzo R., 2006, ApJL, 645, L101

Cardelli J. A., Clayton G. C., Mathis J. S., 1989, ApJ, 345, 245

Cepa J., et al., 2003, SPIE, 4841, 1739, SPIE.4841

Cherenkov Telescope Array Consortium T., 2020, arXiv, arXiv:2010.01349

de Menezes R., Amaya-Almazán R. A., Marchesini E. J., Peña-Herazo H. A.,

Massaro F., Chavushyan V., Paggi A., et al., 2020, Ap\&SS, 365, 12

Falomo R., Pian E., Treves A., 2014, A\&ARv, 22, 73

Freudling W., Romaniello M., Bramich D. M., Ballester P., Forchi V., GarcíaDabló C. E., Moehler S., et al., 2013, A\&A, 559, A964
Giommi P., Glauch T., Padovani P., Resconi E., Turcati A., Chang Y. L., 2020a, MNRAS, 497, 865

Giommi P., Padovani P., Oikonomou F., Glauch T., Paiano S., Resconi E., 2020b, A\&A, 640, L4

Hook I. M., Shaver P. A., Jackson C. A., Wall J. V., Kellermann K. I., 2003, A\&A, 399, 469

IceCube Collaboration, 2018, Science, 361, 147

IceCube Collaboration et al., 2018, Science, 361, eaat 1378

Jones D. H., Read M. A., Saunders W., Colless M., Jarrett T., Parker Q. A., Fairall A. P., et al., 2009, MNRAS, 399, 683

Landoni M., Paiano S., Falomo R., Scarpa R., Treves A., 2018, ApJ, 861, 130

Landoni M., Falomo R., Paiano S., Treves A., 2020, ApJS, 250, 37

Lucarelli F., et al., 2019, ApJ, 870, 136

Mannucci F., Basile F., Poggianti B. M., Cimatti A., Daddi E., Pozzetti L., Vanzi L., 2001, MNRAS, 326, 745

Maselli A., Massaro F., D’Abrusco R., Cusumano G., La Parola V., Segreto A., Tosti G., 2015, Ap\&SS, 357, 141

Massaro F., Masetti N., D’Abrusco R., Paggi A., Funk S., 2014, AJ, 148, 66

Massaro F., Landoni M., D’Abrusco R., Milisavljevic D., Paggi A., Masetti N., Smith H. A., et al., 2015, A\&A, 575, A124

Massaro E., Maselli A., Leto C., Marchegiani P., Perri M., Giommi P., Piranomonte S., 2015, Ap\&SS, 357, 75

Padovani P., Giommi P., 1995, ApJ, 444, 567

Padovani P., Resconi E., Giommi P., Arsioli B., Chang Y. L., 2016, MNRAS, 457, 3582

Padovani P., et al., 2017, A\&ARv, 25, 2

Padovani P., Giommi P., Resconi E., Glauch T., Arsioli B., Sahakyan N., Huber M., 2018, MNRAS, 480, 192

Padovani P., Oikonomou F., Petropoulou M., Giommi P., Resconi E., 2019, MNRAS, 484, L104

Paiano S., Landoni M., Falomo R., Treves A., Scarpa R., Righi C., 2017, ApJ, 837, 144

Paiano S., Landoni M., Falomo R., Treves A., Scarpa R., 2017, ApJ, 844, 120

Paiano S., Falomo R., Franceschini A., Treves A., Scarpa R., 2017, ApJ, 851, 135

Paiano S., Falomo R., Treves A., Scarpa R., 2018, ApJ, 854, L32

Paiano S., Falomo R., Treves A., Franceschini A., Scarpa R., 2019, ApJ, 871, 162

Paiano S., Padovani P., Falomo R., Giommi P., Scarpa R., Treves A., 2019, ATel, 13202

Paiano S., Falomo R., Padovani P., Giommi P., Gargiulo A., Uslenghi M., Rossi A., et al., 2020, MNRAS, 495, L108

Paiano S., Falomo R., Treves A., Scarpa R., 2020, MNRAS, 497, 94

Patrignani C., Particle Data Group, 2016, ChPhC, 40, 100001, p. 528

Peña-Herazo H. A., Marchesini E. J., Álvarez Crespo N., Ricci F., Massaro F., Chavushyan V., Landoni M., et al., 2017, Ap\&SS, 362, 228

Petropoulou M., Dimitrakoudis S., Padovani P., Mastichiadis A., Resconi E., 2015, MNRAS, 448, 2412

Petropoulou M., Oikonomou F., Mastichiadis A., Murase K., Padovani P., Vasilopoulos G., Giommi P., 2020, ApJ, 899, 113

Righi C., Tavecchio F., Inoue S., 2019, MNRAS, 483, L127

Sandrinelli A., Treves A., Falomo R., Farina E. P., Foschini L., Landoni M., Sbarufatti B., 2013, AJ, 146, 163

Sbarufatti B., Treves A., Falomo R., 2005, ApJ, 635, 173

Sbarufatti B., Treves A., Falomo R., Heidt J., Kotilainen J., Scarpa R., 2006, AJ, 132, 1

Shaw M. S. et al., 2013, ApJ, 764, 135

Schlafly E. F., Finkbeiner D. P., 2011, ApJ, 737, 103

Schneider, A., 2019, PoS, ICRC2019, 1004

Stettner, J., 2019, PoS, ICRC2019, 1017

Tody D., 1986, SPIE, 627, 733, SPIE..627

Tody D., 1993, ASPC, 52, 173, ASPC...52

Urry C. M., Padovani P., 1995, PASP, 107, 803

${ }^{6}$ http://web.oapd.inaf.it/zbllac/ 

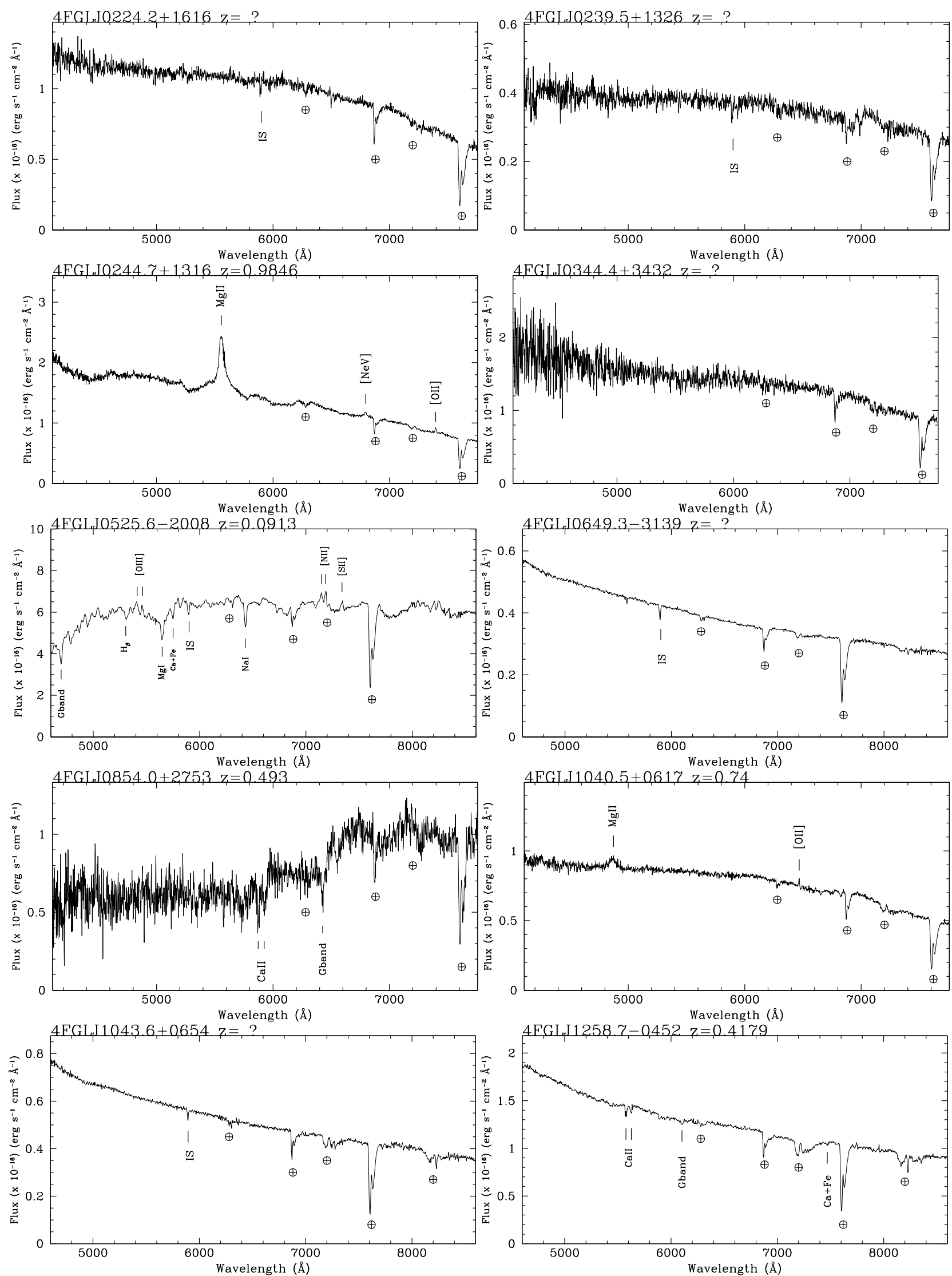

Figure 1. Flux calibrated and dereddened spectra of the neutrino candidate blazars obtained at GTC and VLT. The main telluric bands are indicated by $\oplus$, the absorption features from interstellar medium of our galaxies are labelled as IS (Inter-Stellar). 

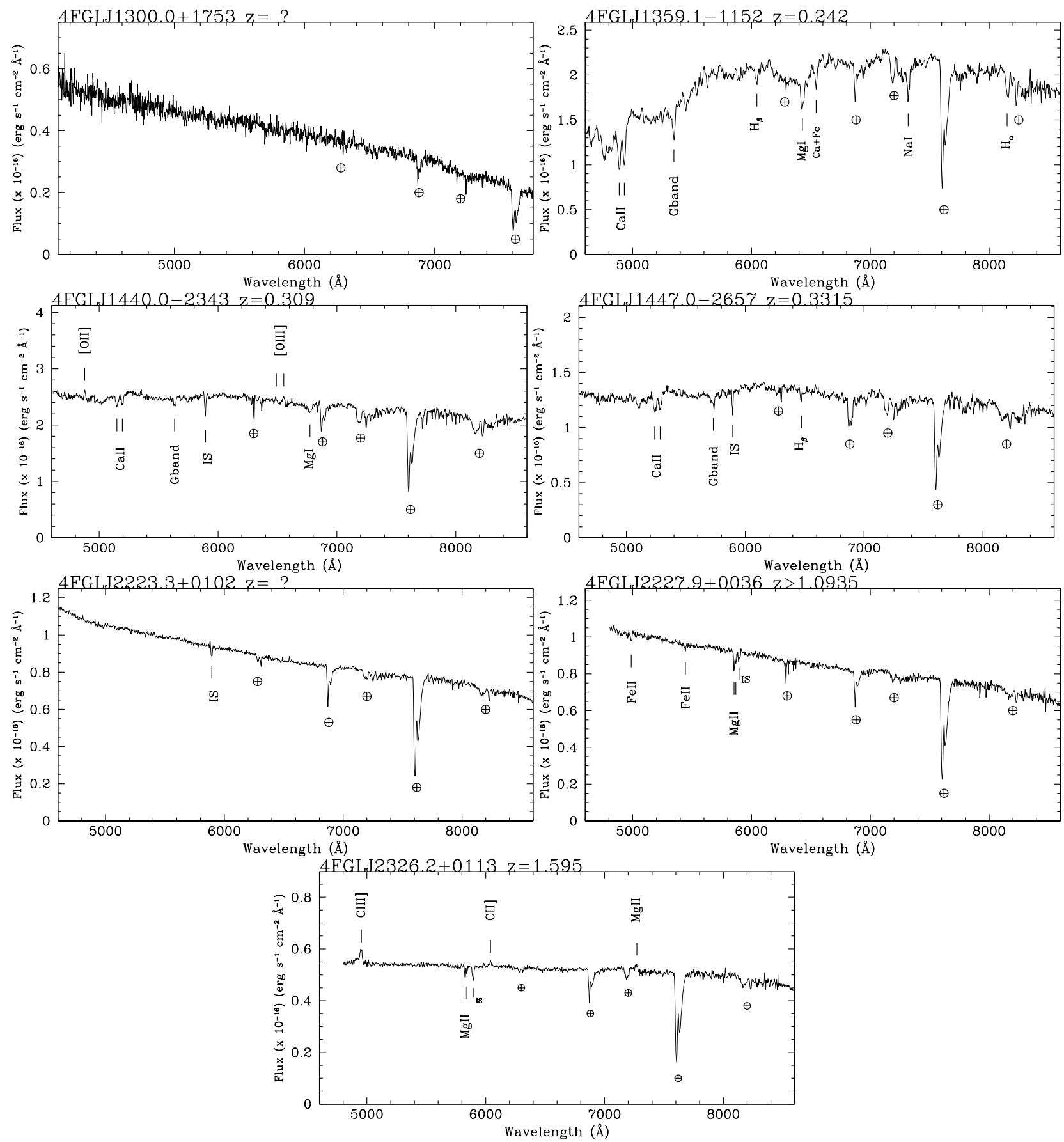

Figure 1. - Continued

\section{APPENDIX A: SPECTROSCOPY OF ADDITIONAL SOURCES}

Here we focus on the seven sources that were considered in a preliminary list of possible counterparts of the neutrino events. They have been eliminated from the list of of 47 objects of Giommi et al 2020 (see Table 1) because either they do not belong to the 4FGL catalogue or the angular separations with respect to the neutrino events do not fulfill the criteria given in the Section 2. The list of these additional sources is given in Table A1, the journal of observations in Table A2 and the spectral properties and redshifts are reported in Table A3. Three objects have a quasar-like line spectrum
(FL8YJ1302.5+2037, 4FGL J2133.0+2610, 4FGL J2311.7+2604) and are among the most distant ones discussed in this paper. The other four appear bona fide BLL. The redshift of two derives from emission lines (4FGL J1339.0-2400 and 4FGL J2325.6+1644). Finally, for one object (4FGL J2255.1+241) a robust lower limit to the redshift is derived from an $\mathrm{Mg}$ II intervening absorption (see also Paiano et al. 2019b).

\section{A1 Notes on individual sources}

FL8YJ1302.5+2037: The object is reported in the FL8Y $\gamma$-ray catalogue, but not in the 4FGL. In our spectrum, we clearly detect 

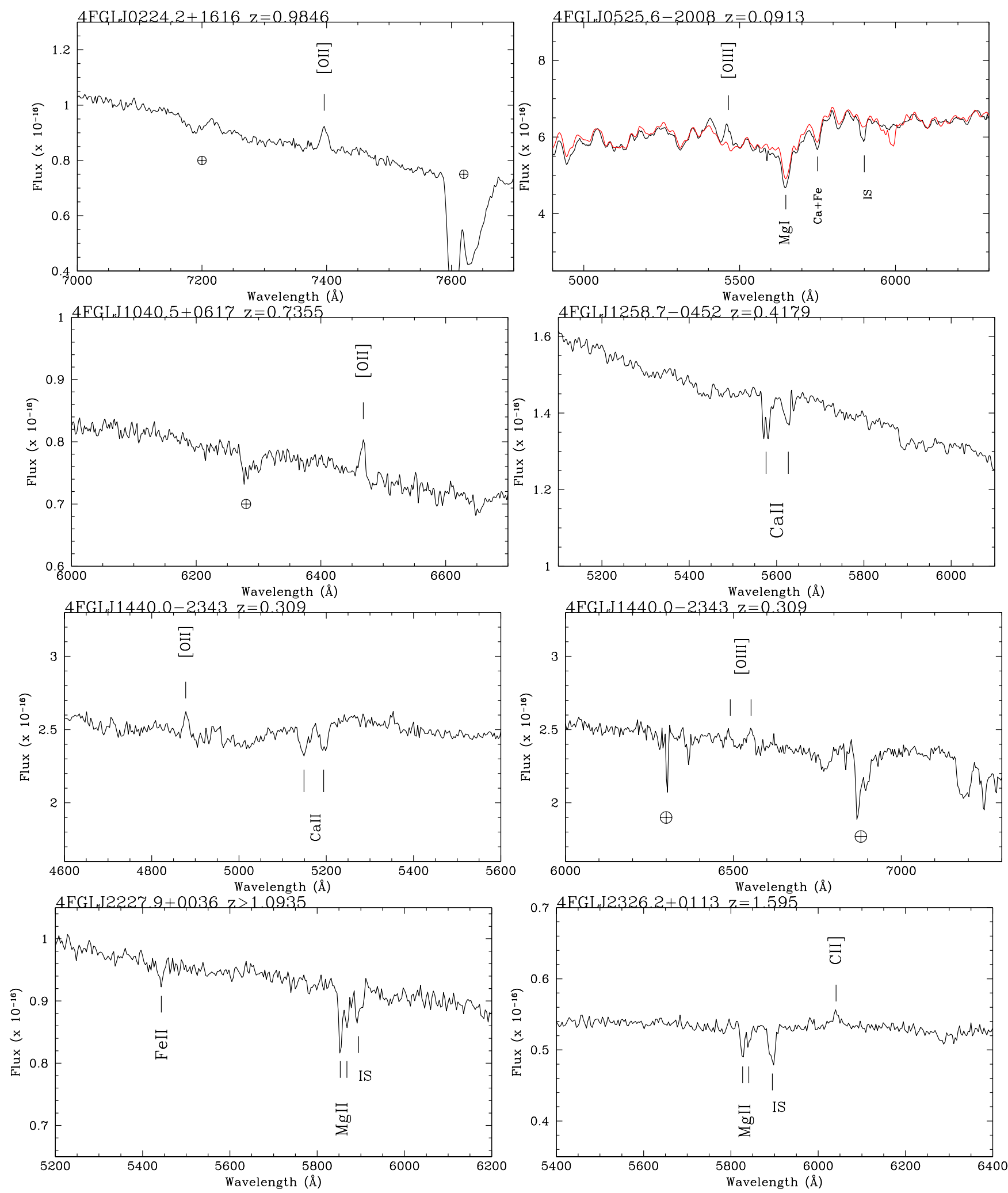

Figure 2. Some examples of close-ups around the detected spectral lines of the spectra of the neutrino candidate blazars obtained at GTC and VLT. Main telluric bands are indicated by $\oplus$, spectral lines are marked by line identification. The red solid line for the source 4FGL J0525.6-2008 is the fit of the spectrum as combination of an elliptical galaxy spectrum and a power law continuum (see Fig. 3 ): this allows us to highlight the detection of the [OIII] 5007 emission line. 

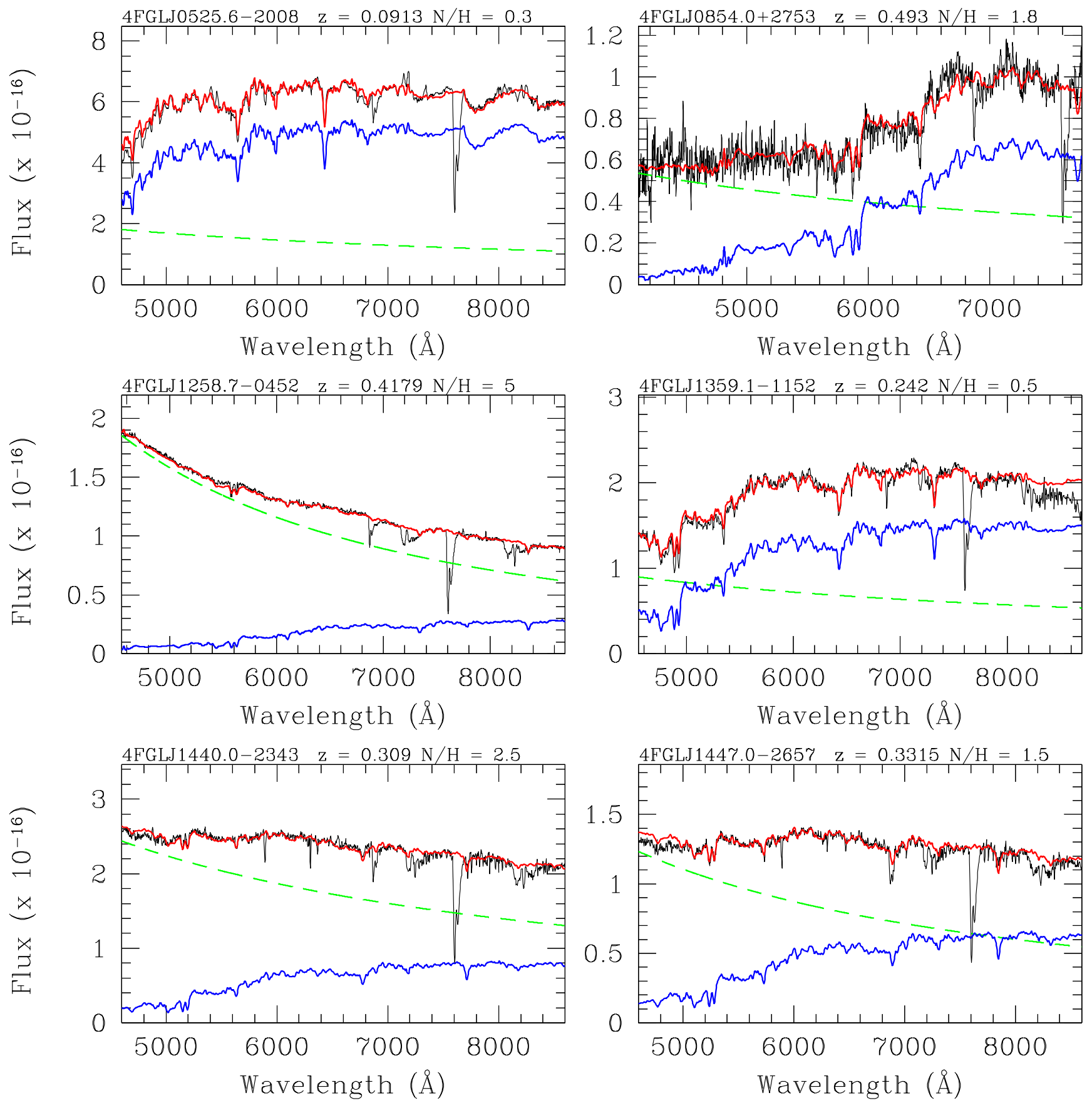

Figure 3. Spectral decomposition of the observed optical spectrum (black line) of some target of our sample into a power law (green dashed line ) and a template of elliptical for the host galaxy (blue line). The fit is given by the red solid line (see text in Section 4 for details). On each panel the nucleus to host ratio is given.

strong and broad (EW ranging from $\sim 33$ to $127 \AA$ ) emission lines attributed to C IV, He II, C III] and Mg II (see Tab. A.3) that lead to a redshift $\mathrm{z}=1.738$. The target can be classified as a quasar.

4FGL J1339.0-2400: We obtained a high quality ( $\mathrm{S} / \mathrm{N} \sim 220)$ spectrum of the target $(g=18.9)$ which exhibits faint emission lines attributed to $[\mathrm{O} \mathrm{II}],[\mathrm{Ne} \mathrm{III}]$ and $[\mathrm{O} \mathrm{III}]$. This yields a redshift of
$\mathrm{Z}=0.655$, confirming the proposed uncertain redshift by Hook et al. (2003).

4FGL J1455.0+0247: This source was observed by Sandrinelli et al. (2013) and by the SDSS and no emission/absorption lines were detected. Our $(\mathrm{S} / \mathrm{N} \sim 65)$ spectrum of the source $(\mathrm{g}=20.2)$ is still 
featureless and from the estimated minimum $\mathrm{EW} \sim 0.50 \AA$ A we can set a redshift lower limit $\mathrm{z}>0.65$.

4FGL J2133.0+2610: Our spectrum exhibits a prominent broad emission line (see Tab. A3) that if attributed to Mg II yields a redshift $\mathrm{z}=1.139$. In addition we detected two absorption doublets at 5340,5369 $\AA$ and 5774,5788 $\AA$ due to a Fe II and Mg II intervening system respectively, corresponding to a $\mathrm{z}=1.065$.

4FGL J2255.1+2411: The source has been also recently proposed as a possible counterpart of five ANTARES neutrino events with energy ranging from 3 to $40 \mathrm{TeV}$ (see Aublin 2019; Paiano et al. 2019b; Giommi et al. 2020a). In our very high quality ( $\mathrm{S} / \mathrm{N}=550)$ spectrum, we detect a Mg II intervening system at 5209,5222 $\AA$ allowing us to set the spectroscopic redshift lower limit $\mathrm{z} \geq 0.863$. In previous spectra (Shaw et al. 2013; Massaro et al. 2015, and the SDSS survey) no emission or absorption lines are identified but the feature at 5209,5222 $\AA$ is visible.

4FGL J2311.7+2604: In our spectrum we clearly detected broad emission lines attributed to $\mathrm{C} \mathrm{IV}$, He II, CIII], and Mg II (see Tab. A3). This implies a redshift of $\mathrm{z}=1.7425$, confirming the value of the SDSS spectrum.

4FGL J2325.6+1644: In the literature, Massaro et al. (2015) report an optical spectrum of modest $\mathrm{S} / \mathrm{N}$ without evident features. In our spectrum, we detect two narrow emission lines due to forbidden transitions attributed to [O II] and [O III] (see Tab. A3), yielding $\mathrm{z}=0.4817$. 
Table A1. Additional Neutrino candidate blazars.

\begin{tabular}{lllllllll}
\hline Object Name & Counterpart & IC event & $\begin{array}{l}\text { RA } \\
(J 2000)\end{array}$ & $\begin{array}{l}\text { DEC } \\
(\text { J2000 })\end{array}$ & Magnitude & $v_{\text {peak }}^{\text {syn }}$ & Redshift & Reference \\
\hline FL8Y J1302.5+2037 & CRATES J130215+203911 & IC-151017A & $13: 02: 14.6$ & $+20: 39: 18.6$ & 20.1 & 12.5 & $?$ \\
\hline 4FGL J1339.0-2400 & 5BZU J1339-2401 & IC-131202A & $13: 39: 01.7$ & $-24: 01: 14.0$ & 18.2 & 14.1 & $0.665 ?$ & NED \\
\hline 4FGL J1455.0+0247 & 5BZB J1455+0250 & IC-111201A & $14: 55: 07.4$ & $+02: 50: 40.1$ & 19.7 & 14.3 & $?$ & $*$ \\
\hline 4FGL J2133.0+2610 & NVSS J213252+261143 & IC-150714A & $21: 32: 52.9$ & $+26: 11: 43.8$ & 20.5 & 13.0 & $?$ & $*$ \\
\hline 4FGL J2255.1+2411 & MG3 J225517+2409 & IC-100608A & $22: 55: 15.3$ & $+24: 10: 11.3$ & 18.6 & 14.7 & $?$ \\
4FGL J2311.7+2604 & MG3 J231144+2604 & IC-100608A & $23: 11: 45.8$ & $+26: 04: 47.8$ & 21.2 & 13.0 & 1.74 & SDSS \\
\hline 4FGL J2325.6+1644 & NVSS J232538+164641 & IC-140522A & $23: 25: 38.1$ & $+16: 46: 42.2$ & 18.2 & 15.3 & $?$ & ZBLLAC \\
\hline
\end{tabular}

Notes. Column 1: Fermi name of the target in the 4FGL catalogue; Column 2: Counterpart name of the $\gamma$-ray source; Column 3: IceCube track; Column 4: Angular separation (degrees) between the target and the centroid of the IceCube track; Column 5 - 6: Right ascension and declination of the optical counterpart; Column 7: g magnitude from SDSS survey and PANSTARRs; Column 8: Frequency of the synchrotron peak ( $v_{\text {peak }}^{s y n}$ ); Column 9: Redshift from the literature.

Table A2. Journal of the observations of additional neutrino candidate blazars.

\begin{tabular}{lllllll}
\hline Object Name & E(B-V) & Telescope & Date & $\begin{array}{l}\mathrm{t}_{\text {Exp }} \\
(\mathrm{s})\end{array}$ & $\begin{array}{l}\text { seeing } \\
(")\end{array}$ & Air Mass \\
\hline FL8Y J1302.5+2037 & 0.02 & GTC & 02 February 2020 & 6600 & 2.0 & 1.02 \\
4FGL J1339.0-2400 & 0.07 & VLT & 02 February 2020 & 5400 & 0.8 & 1.09 \\
4FGL J1455.0+0247 & 0.04 & VLT & 21 March 2020 & 5400 & 1.4 & 1.31 \\
4FGL J2133.0+2610 & 0.11 & GTC & 12 October 2019 & 6600 & 1.7 & 1.32 \\
4FGL J2255.1+2411 & 0.06 & GTC & 04 October 2019 & 21600 & 0.9 & 1.10 \\
4FGL J2311.7+2604 & 0.05 & GTC & 21 October 2019 & 4500 & 2.0 & 1.14 \\
4FGL J2325.6+1644 & 0.03 & GTC & 09 October 2019 & 3000 & 1.0 & 1.13 \\
\hline
\end{tabular}

Notes. Column 1: Name of the target ; Column 2: E(B-V) taken from the NASA/IPAC Infrared Science Archive (https://irsa.ipac.caltech.edu/applications/DUST/); Column 3: Telescope used for the observation; Column 4: Date of the observation; Column 5: Total integration time (sec); Column 6: Average seeing during the observation $(\operatorname{arcsec})$.

Table A3. Spectral properties of the additional neutrino candidate blazars.

\begin{tabular}{lllllll}
\hline Object Name & $\mathrm{g}$ & $\mathrm{S} / \mathrm{N}$ & $\begin{array}{l}\mathrm{EW}_{\text {min }} \\
(\AA)\end{array}$ & $\mathrm{z}$ & Line type & $\alpha$ \\
\hline FL8Y J1302.5+2037 & 20.1 & 35 & - & 1.738 & $\mathrm{e}, \mathrm{i}$ & $1.2 \pm 0.2$ \\
4FGL J1339.0-2400 & 18.9 & 220 & - & 0.655 & $\mathrm{e}$ & $0.3 \pm 0.1$ \\
4FGL J1455.0+0247 & 20.2 & 65 & 0.50 & $>0.65$ & $\mathrm{~h}$ & $0.0 \pm 0.1$ \\
4FGL J2133.0+2610 & 20.3 & 20 & - & 1.139 & $\mathrm{e}, \mathrm{i}$ & $1.2 \pm 0.3$ \\
4FGL J2255.1+2411 & 17.5 & 550 & 0.10 & $>0.863$ & $\mathrm{i}$ & $0.95 \pm 0.1$ \\
4FGL J2311.7+2604 & 21.6 & 10 & - & 1.7425 & $\mathrm{e}$ & $1.1 \pm 0.1$ \\
4FGL J2325.6+1644 & 18.7 & 100 & - & 0.4817 & $\mathrm{e}$ & $0.9 \pm 0.1$ \\
\hline
\end{tabular}

Notes. Column 1: Name of the target; Column 2: Magnitude (g) measured from the acquisition image; Column 3: Median S/N of the spectrum; Column 4: Minimum equivalent width $\left(\mathrm{EW}_{\min }\right)$ derived in the 5500 - $6500 \AA$ range (the measure is provided only in case of featureless spectrum); Column 5: Redshift; Column 6: Type of detected line to estimate the redshift: $e=$ emission line, $g=$ galaxy absorption line, $i=$ intervening absorption assuming Mg II $2800 \AA$ identification, $h=$ lower limit derived on the lack of detection of host galaxy absorption lines assuming a BLL elliptical host galaxy with M(R) $=-22.9$ (see details in Paiano et al. 2017a). Column-7: Spectral index $\alpha$ of the continuum described by a power law $\mathrm{F}_{\lambda} \sim \lambda^{-\alpha}$ 
Table A4. Properties of the emission lines.

\begin{tabular}{|c|c|c|c|c|c|}
\hline Object Name & $\mathrm{z}$ & $\begin{array}{l}\lambda \\
(\AA)\end{array}$ & $\begin{array}{l}\text { EW } \\
(\AA)\end{array}$ & Line ID & $\begin{array}{l}L \text { (line) } \\
\left(\mathrm{erg} \mathrm{s}^{-1}\right)\end{array}$ \\
\hline \multirow[t]{4}{*}{ FL8Y J1302.5+2037 } & 1.738 & 4242 & $127 \pm 5$ & C IV 1549 & $5.6 \times 10^{44}$ \\
\hline & & 4491 & $33 \pm 2$ & He II 1640 & $4.7 \times 10^{43}$ \\
\hline & & 5226 & $95 \pm 3$ & C III] 1909 & $1.5 \times 10^{44}$ \\
\hline & & 7666 & $19: \pm 5$ & Mg II 2800 & $1.2 \times 10^{43} *$ \\
\hline \multirow[t]{3}{*}{ 4FGL J1339.0-2400 } & 0.655 & 6168 & $1.3 \pm 0.3$ & [O II] 3727 & $4.0 \times 10^{41}$ \\
\hline & & 6403 & $0.50 \pm 0.2$ & [Ne III] 3869 & $1.5 \times 10^{41}$ \\
\hline & & 8287 & $2.2 \pm 0.2$ & [O III] 5007 & $6.2 \times 10^{41}$ \\
\hline 4FGL J2133.0+2610 & 1.139 & 5989 & $50 \pm 3$ & Mg II 2800 & $3.5 \times 10^{43}$ \\
\hline \multirow[t]{4}{*}{ 4FGL J2311.7+2604 } & 1.7425 & 4246 & $155 \pm 6$ & C IV 1549 & $2.0 \times 10^{44}$ \\
\hline & & 4499 & $66 \pm 4$ & He II 1640 & $2.5 \times 10^{43}$ \\
\hline & & 5235 & $16 \pm 2$ & C III] 1909 & $5.6 \times 10^{42}$ \\
\hline & & 7679 & $8: \pm 3$ & Mg II 2800 & $1.9 \times 10^{42 *}$ \\
\hline \multirow[t]{2}{*}{ 4FGL J2325.6+1644 } & 0.4817 & 5522 & $0.70 \pm 0.2$ & [O II] 3727 & $1.0 \times 10^{41}$ \\
\hline & & 7419 & $0.50 \pm 0.2$ & [O III] 5007 & $4.8 \times 10^{40}$ \\
\hline
\end{tabular}

Notes. Column 1: Name of the target; Column 2: Redshift; Column 3: Barycenter of the detected line; Column 4: Measured equivalent width of the line; Column 5: Line identification; Column 6: Line luminosity.

(*) These lines are partially blended with the telluric band. 

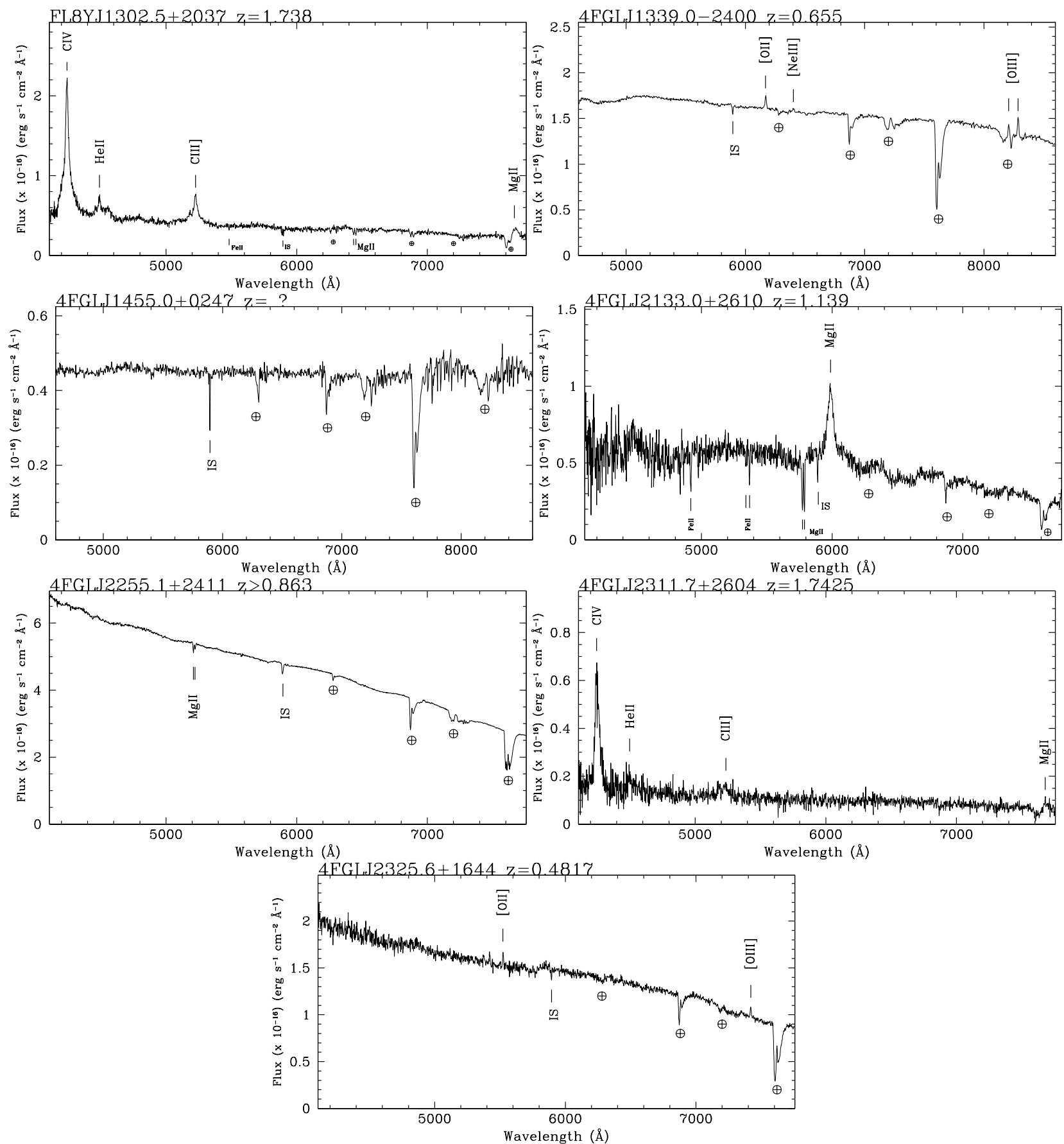

Figure A1. Flux calibrated and dereddened spectra of the neutrino candidate blazars obtained at GTC and VLT. The main telluric bands are indicated by $\oplus$, the absorption features from interstellar medium of our galaxies are labelled as IS (Inter-Stellar). 